\title{
Special Issue: Research on Sexual Violence in the \#MeToo Era: Prevention and Innovative Methodologies
}

\author{
Leah E. Daigle ${ }^{1}$ \\ Accepted: 8 December 2020/ \\ Published online: 4 January 2021 \\ (C) Southern Criminal Justice Association 2021
}

\section{Guest Editor's Introduction}

The study of sexual victimization is not new. Dating back some 70 years, researchers began using self-report surveys to measure a variety of forms of sexual victimization (Fisher, Daigle, \& Cullen, 2010). Indeed, these early efforts by Kirkpatrick and Kanin (1957) demonstrated that $6.2 \%$ of college women had experienced aggressive forceful attempts at sexual intercourse during which either threats or coercive infliction of physical pain were used during the academic year (p. 53). These findings reflect what we consider rape to be today. Despite this early finding, there was not an immediate reaction among researchers, educators, or practitioners to focus more fully on sexual victimization of women. Instead, an influx of research on sexual victimization often occurs during periods where there is public attention on sexual victimization and violence against women. Indeed, during the late 70 s and 80 s several important, largescale studies on the sexual victimization of women were conducted reflecting the women's movement and its recognition that violence against women should not be a private manner, is common, and deserves special investigation (see for example Koss, Gidycz, \& Wisnewski, 1987; Russell, 1982). Of course, research is often not borne out of any particular social movement, but media attention and public awareness has helped bring to light the pervasive problem of sexual victimization.

One such recent movement is the MeToo movement. Although widely popularized in 2017, the MeToo movement originated in 2006 by Tarana Burke. Tarana Burke began using the phrase "me too" on the social networking website Myspace to highlight occurrences of sexual harassment. This original use was focused on women of color as targets of sexual harassment (me too, 2020). The MeToo movement was revitalized when, in 2017, the actress Alyssa Milano used the \#MeToo phrase on Twitter - which went viral. She, along with other actresses, began using the hashtag to publicly discuss

Leah E. Daigle

1daigle@gsu.edu

1 Department of Criminal Justice and Criminology, Faculty Affiliate in Center for Interpersonal Violence, Georgia State University, Atlanta, GA, USA 
their experiences with sexual harassment. The hashtag generated 12 million uses during the first 24 hours after it began trending (as cited by Hoffman, this issue). Along with this awareness, the me too movement organization provides assistance to survivors of sexual harassment and works to hold offenders accountable (me too, 2020).

Given the widespread attention the \#MeToo received coupled with the arrests of several high-profile Hollywood figures such as Harvey Weinstein and Bill Cosby, it would not be surprising to see another wave of research on sexual victimization that focuses on the link of awareness and how social media campaigns influence occurrence, reporting, and responses to sexual victimization. To that end, this special issue highlights research centered on how the \#MeToo movement shapes sexual victimization experiences, and how innovative methodologies may be employed to more fully capture the range of sexual victimization experiences. The first four articles of the special issue examine how college students perceive sexual victimization, how aware they are of sexual victimization and their willingness to use bystander intervention skills, and how disclosure of sexual victimization is influenced by the \#MeToo movement.

In the first article, Acquaviva and colleagues use interview data from college students and alumni to examine sources of awareness of sexual assault cases in the media and perceptions of victim believability based on the accounts they read or heard about. Almost two-thirds of their participants reported learning about sexual assault through the media, and almost all of the participants were able to reference a specific sexual assault case that occurred between 2017 and 2018. About 7 in 10 respondents reported believing the allegations. This piece indicates that the media may be a source of knowledge about sexual assault and that the media has a responsibility for fair reporting. The second article explores how participation in sexual violence campus programming influences bystander intervention (Azimi et al.). The authors find that attending campus events designed to raise awareness of sexual violence was associated with a reduction in the odds of engaging in bystander intervention in a risky situation, while experiencing sexual victimization increased the odds. Importantly, the programming types that were measured were awareness-based, such as Walk a Mile and Ignite the Night, rather than formal campus-sponsored programming, which suggests that the type of program or exposure to knowledge of sexual victimization needs to be specifically examined in other research. In this study, bystander intervention training was unrelated to bystander intervention, suggesting that prospective research needs to be conducted that can clearly delineate time-order - that training or participation precedes bystander intervention. Another examination of participation in awareness campaigns was performed by Hoffman in the third article. The author examines factors that influence participation in the \#MeToo movement either by posting the \#MeToo hashtag on social media accounts, signing a \#MeToo petition, or attending a \#MeToo protest or rally. Understanding engagement in social movements and digital activism and the factors that influence this engagement are important, especially given how participating may influence perceptions of victims and intervention likelihood. Insomuch that victimization is linked to participation (as found by Hoffman), it may also structure revictimization risk in that persons who receive positive feedback or have a positive experience through their engagement may find social support that could disrupt the victimization-revictimization nexus. Further, Hoffman finds that women and sexual minorities are more likely to engage in the \#MeToo movement, whereas those students affiliated with Greek fraternities or sororities were less likely to 
participate. These differences suggest that additional outreach to these groups is necessary. Connected to these works is the fourth article by Palmer and colleagues, in which they investigate prevalence and disclosure of sexual victimization from two time periods - one before the second wave of the \#MeToo movement in March 2017 and one delivered in March 2019. The authors also assessed students' belief that the \#MeToo movement affected how they think about past experiences that have happened to them. The authors' findings improve our understanding of the \#MeToo movement and its possible influences on experience and perception. They find an increase in disclosure among Black students from 2017 to 2019, which could indicate the power of the \#MeToo movement and its particular focus on persons of color and underrepresented groups. They also found that people who believed \#MeToo affected their perceptions of previous experiences were more likely to indicate past experiences with unwanted sexual activity. Although impossible to discern, the \#MeToo movement may be helping victims contextualize and define their own experiences, which could be an important step in healing and receiving resources for them.

The next set of articles combine to improve our understanding of sexual victimization that occurs outside of the college environment and instead in places that are likely to bring together strangers in close contact where they are not necessarily able to leave, even if threatened. In the fifth article, Ceccato and Loukaitou-Sideris provide an overview of a cross-national large-scale research effort on understanding sexual harassment that occurs in public transit environments. Their research team conducted studies of university students in 18 difference cities across six different continents, all with the goal of understanding the prevalence of sexual harassment in diverse transit environments. Particularly salient is their discussion about the methodological challenges they faced in developing and administering a survey across these various locales. They make note that a one-size fits all approach to curbing sexual harassment in transit environments is unlikely to be successful given the cultural contexts in which it occurs. Another understudied location of sexual victimization is airplanes. Much like with public transit, an airline passenger may perceive few options when facing threat during a flight. Despite the seeming potential risk for sexual victimization to occur, systematic data collection has not been conducted. As noted by Lucas in the sixth article, the FBI has jurisdiction over reported incidents of sexual victimization taking place on commercial aircrafts, and while there has been an increase in the number of cases reported from 2014 to 2017 , these data only reflect reported incidents and do not provide details on the context in which they occur. To investigate the specific details surrounding in-flight sexual victimization, Lucas performs content analysis of media reports from 2006 through 2020. The author identified 123 cases of in-flight sexual victimization involving mostly female victims and male offenders who fondled or groped their victim. Most incidents involved offenders who were seated directly beside their victim, underscoring the difficulty of escape or avoidance. Although most flights are free from sexual victimization, the author's findings reveal the unique nature of flights and how they may influence the occurrence of and response to sexual victimization.

The final two articles are methodological in nature, and both investigate how measuring sexual victimization, although done via self-report surveys for some 70 years, is still difficult. Indeed, even minor differences in measurement strategy have been shown to produce sometimes wide discrepancies in prevalence. In the seventh article, Anderson and colleagues evaluate two surveys designed to measure sexual victimization: the Post- 
Refusal Sexual Persistence Scale (PRSPS) and the tactic-first Sexual Experiences Survey (SES). Tactic-first questionnaires provide items in which the possible tactic (e.g., force) used to coerce sexual behavior is in the first part of the item and then the behavior that was attempted or completed (e.g. sex) is in the second part of the item. Behavior-first items, on the other hand, provide the respondent with questions where the behavior is first identified and then the tactic. Although subtle, the authors find support that providing tactics first produces higher prevalence estimates. In the last article, Anderson, Namie, and Goodman again use two common measures of sexual victimization to investigate their validity for sexual minorities and men. They find convergent validity in that scores generated from the SES-SFV and the PRSPS-V were strongly correlated, yet weakest for heterosexual men. They also investigate differential validity and find that when examining scores on the SESSFV and PRSPS-V and their correlations to rape empathy, the strength of the correlations differed by sexual identity and gender. Considered together, these two articles' findings further the argument that good measurement is key in truly understanding prevalence and that measures need to be developed for and validated on specific groups.

Considered together, the articles in this special issue further our understanding of how social movements and digital activism may shape the landscape of sexual victimization. Further, innovative methodologies focused on specific forms and contexts in which sexual victimization occur are explored. The findings can help guide the next set of researchers in developing and implementing measurement tools in general and in including questions specific to social movements and the context in which sexual victimization occurs.

\section{References}

Fisher, B. S., Daigle, L. E., \& Cullen, F. T. (2010). Unsafe in the ivory tower. Thousand Oaks, CA: Sage Publications.

Kirkpatrick, C., \& Kanin, E. J. (1957). Male sexual aggression on a university campus. American Sociological Review, 22, 52-58.

Koss, M. P., Gidycz, C. A., \& Wisnewski, N. (1987). The scope of rape: Incidence and prevalence of sexual aggression in a national sample of higher education students. Journal of Consulting and Clinical Psychology, 55, 162-170.

me too. (2020). https://metoomvmt.org/get-to-know-us/history-inception/. Accessed 3 Dec 2020.

Russell, D. E. H. (1982). The prevalence and incidence of forcible rape and attempted rape of females. Victimology: An International Journal, 7, 81-93.

Publisher's Note Springer Nature remains neutral with regard to jurisdictional claims in published maps and institutional affiliations.

Dr. Leah E. Daigle is Professor in the Department of Criminal Justice in the Andrew Young School of Policy Studies at Georgia State University. Her most recent research has centered on recurring victimization, sexual victimization of college women, and the development and continuation of victimization across the life course. She is coauthor of Criminals in the Making: Criminality Across the Life Course (2nd ed.) and Unsafe in the Ivory Tower: The Sexual Victimization of College Women, which was awarded the 2011 Outstanding Book Award by the Academy of Criminal Justice Sciences, and author of Victimology: A Text/Reader (2nd ed.) and Victimology: The Essentials (2nd ed.). Her research has also appeared in peer-reviewed journals including Justice Quarterly, Victims and Offenders, The Journal of Quantitative Criminology, and The Journal of Interpersonal Violence. She was awarded the 2014 Andrew Young School of Policy of Studies Excellence in Teaching Award and the 2020 Division of Victimology of the American Society of Criminology Bonnie S. Fisher Victimology Career Award. 